was eleven days, in two it was twelve days, in one it was probably eleven or twelve days.

In eleven cases the eruption was seen two days after the first feeling of discomfort; in twelve, three days after ; in eight, four days after; in one, in less than two days ; in two cases the date was not fixed, and in one case there was no eruption.

Of those cases whose duration could be accurately determined varioloid lasted from 13 to 29 days, there being one case continuing each of the following periods $13,16,17,19,20,21,2 \overline{3}$ and 31 days, and two cases 15 days. The cases of variola which recovered lasted one each during $31,32,41,45,56$ and 60 days two cases each 27, 40 and 42 days. The fatal cases died, one in less than five days, one in $8,9,14$ and 16 days, and two in 15 days.

Except in the hæmorrhagic variety. which is much more quickly fatal, the date of greatest mortality and danger is between the fourteenth and sixteenth days.

Chills which are mentioned as a prominent symptom at the commencement were absent in many cases, and in most of the others were so slight as not to be mentioned by the patient unless questioned in regard to that point. Later in the disease during the suppura tion and still more during desiccation there were frequently severe chills.

In only one case which recovered, was there entire absence of salivation; usually this symptom was well marked.

In three cases there was pulmonary complication; in only one showing decided signs of pneumonia; the other two being probably only bronchitis and pleurisy.

Vomiting at the beginning of the disease occurred in both severe and mild cases, being, however, rather more protracted in severe cases. It did not seem to have much value for prognosis.

In two cases there was a pustule on the conjunctiva and in one case during the occurrence of abscesses in other parts there was a collection of pus under the conjunctiva.

In four cases variola occurred after reported vaccination. In one of these the patient said the vaccina tion did not take well, in another the scars could not be distinguished with certainty, in two cases the examination was not accurate enough to state positively what was the condition of the scars. The other cases of variola had never been vaccinated. A winter's experience in a small-pox hospital during an epidemic would probably cure ove of any prejudice against vaccination. In only two cases of variola was it probable that vaccination may have been well performed, and both of these were mild cases and recovered. Comparing the severity and duration of the disease in the 15 who had been vaccinated and the 19 who had not, or had been only imperfectly protected, there can be no hesitation in regard to a decision in favor of vaccination.

A few reflections occur, as I now look over this paper, in regard to the improved sanitary condition and care of the City as compared with 1870 . Then, a patient with the scabs still on his face could ride in a public car without giving rise to any inquiry, scarcely to comment. A patient now would not be sent to the hospital alone, nor would such an one wander about the streets trying to find it, as in case VI. And it would not now be possible for 80 many to take care of their friends until they died with the disesse, if doctors did their duty.

\section{THE TREATMENT OF PULMONARY TUBERCU- LOSIS BY THE SUBCUTANEOUS USE OF THE CHLORIDE OF GOLD AND SODIUM WITH THE IODIDE OF MANGANESE.}

BY WM. S. BOARDMAN, M.D., Boston.

We have read and beard so much about new remedies and modificatious of new remedies for the treatment of phthisis, that now to many the subject fails to excite an interest.

The methods of treatment are too numerous to men. tion. One is almost startled by the list of drugs and methods proposed, in reading Solis-Cohen's article on "'Tuberculosis," in " Hare's System of Pructical Therapeutics." Yet there must be something beneficial in these remedies, even if there is nothing specific.

The brilliant methods of Koch, dimmed by the noxious elements in his tuberculin and brought to a glow again by the researches of Klebs and Hunter, are undoubtedly more for good than anything hitherto proposed. Although we seem so near the desired result, it has not yet been obtained in the ideal that was first conceived; nevertheless, it behooves us to make the most of what we have, while striving for something better.

From the earliest time, the attempt has always been made to combat the marasmus of phthisis by dietetic and hygienic measures. How slow and uncertain a procedure it has been is only too well known to us all. If in any way we can aid the process of nutrition and maintain or increase the strength of the patient, a great deal will be done towards staying the disease, if not directly tending to a cure.

One mode of treatment that has been tried considerably yet spoken of by comparatively few writers, is the subcutaneous use of a solution in glycerine, of the chloride of gold and sodium with the iodide of manganese. In March, 1891, an article in the Medical Record, by Dr. J. B. White, extolled this combination as a treatment for phthisis, and cited a number of cases to show the beneficial results obtained. Another article in September, 1892, by the same author, appeared to supplement and confirm the results of the first paper, being written in a very earnest and pleasing manner. As the combination of the chloride of gold and sodium with the iodide of manganese, is, under ordinary circumstances, likely to result in the formation of a precipitate, the author of the above-mentioned articles was asked to state the method of preparing the solution. Since the process was not given and the injection fluid could only be obtained in New York, it was thought to be of sufficiently scientific interest to the profession to have an analysis of Dr. White's preparation; and Mr. Wm. C. Durkee, Ph.G., kindly consented to undertake the chemical work with this result.

"The proximate chemical examination of the injection fluid is as follows. It has a dark-amber color, with a specific gravity of 1,260, a styptic taste and a syrupy consistency.

"According to Dr. J. Blake White's statement, each drop should contain the equivalent of one-fiftieth of a grain of the chloride of gold and sodium, and onefiftieth of a grain of the iodide of mangauese, that is, each drop should represent one-twenty-fifth of a grain of the combined salts.

"He does not mention the method of obtaining the drop, whether from the bottle or a medicine-dropper; 
TREATMENT WITH SUBCUTANEOUS INJECTIONS OF GOLD AND MA

\begin{tabular}{|c|c|c|c|c|}
\hline $\begin{array}{l}\text { No. Sex. Age. } \\
\text { Social Condition. } \\
\text { Occupation. } \\
\text { Family History. }\end{array}$ & $\begin{array}{l}\text { Patient's History. } \\
\text { Physical Examination at Beginning of Treatment. }\end{array}$ & $\begin{array}{l}\text { First Treatment. } \\
\text { Weight. } \\
\text { Sputum. }\end{array}$ & $\begin{array}{l}\text { Last Treatment. } \\
\text { Weight. } \\
\text { Sputum. }\end{array}$ & $\begin{array}{r}\text { Subcus } \\
\text { Total Amc } \\
\text { Largest D }\end{array}$ \\
\hline $\begin{array}{c}\text { I. } \\
\text { Female. } \\
20 \text { years. } \\
\text { Single. } \\
\text { Home-work. } \\
\text { Mother phthisical, } \\
\text { one brother died } \\
\text { of phthisis. }\end{array}$ & $\begin{array}{l}\text { Has not felt well for } 6 \text { months. Cough for } 3 \text { months. Loss of flesh } \\
\text { and strength. Sweats considerably by day. No night sweats. Menses } \\
\text { regular. } \\
\text { Considerable emaciation. Hectic flush. Right clavicle more prom- } \\
\text { inent than the left. No dulness. Respiration roughened nearly } \\
\text { everywhere. A few fine moist râles at the left apex. Medium moist } \\
\text { râles at the right apex anterior. }\end{array}$ & $\begin{array}{l}\text { Feb. 6, } 1893 . \\
111 \text { pounds. } \\
3 \text { ifss-iii. } \\
\text { No tubercle bacilli } \\
\text { found. } \\
11 \text { A. M. } \\
\text { Pulse } 76 . \\
\text { Temp. } 99^{\circ} . \\
\text { Resp. 31. }\end{array}$ & $\begin{array}{l}\text { June } 13,1893 . \\
108 \text { pounds. } \\
\text { (Did weigh } 117 \text { lbs. } \\
\text { after } 34 \text { subcus., } \\
\text { May 6th.) } \\
\text { 3 i-iss. } \\
\text { Tubercle bac illi } \\
\text { fairly numerous. } \\
12.30 \text { P. M. } \\
\text { Pulse } 68 . \\
\text { Temp. } 99.3^{\circ} . \\
\text { Resp. } 35 .\end{array}$ & $\begin{array}{c}60 \\
112 \text { drop } \\
6 \text { drops }\end{array}$ \\
\hline $\begin{array}{c}\text { II. } \\
\text { Female. } \\
\text { 27 years. } \\
\text { Single. } \\
\text { Parlor-girl. } \\
\text { One sister died of } \\
\text { phthisis. }\end{array}$ & $\begin{array}{l}\text { Pneumonia } 2 \text { years ago; never fully regained her health. Hoarse } \\
\text { since last fall. No cough. No expectoration. No hæmoptysis. No } \\
\text { sweats. Loss of flesh and strength. Menses regular (1 day, scanty). } \\
\text { Variable appetite. } \\
\text { Respiration roughened everywhere. Fine moist râles over right } \\
\text { and left upper backs. Ulceration at middle of left vocal cord. }\end{array}$ & $\begin{array}{l}\text { Feb. 17. } \\
115 \text { pounds. } \\
\text { No sputum. } \\
\text { (Former weight 13n } \\
\text { pounds.) } \\
\text { 11 A. M. } \\
\text { Pulse 96. } \\
\text { Temp.98.8. } \\
\text { Resp. 21. }\end{array}$ & $\begin{array}{l}\text { April } 26 . \\
111 \text { s pounds. } \\
\text { No sputum. } \\
12 \text { M. } \\
\text { Pulse 84. } \\
\text { Temp. } 99^{\circ} \text {. } \\
\text { Resp. 21. }\end{array}$ & $\begin{array}{l}21 \\
29 \text { drops } \\
5 \text { drops. }\end{array}$ \\
\hline $\begin{array}{l}\text { III. } \\
\text { Female. } \\
\text { 19 years. } \\
\text { Single. } \\
\text { Paper-box maker. } \\
\text { P aren ts died of } \\
\text { phthisis. }\end{array}$ & $\begin{array}{l}\text { Cough for } 6 \text { months. Occasional night sweats. Fairly strong. } \\
\text { Menses every } 3-4 \text { weeks, and scanty. Appetite poor. } \\
\text { Relative dulness at left apex anterior and below left clavicle. } \\
\text { Bronchial respiration at left apex anterior and over upper half of left } \\
\text { front. Few moist râles over upper half left front houghened res- } \\
\text { piration at right apex and over right front. A click heard at right } \\
\text { apex anterior. Harsh respiration and medium moist râles in left } \\
\text { axilla. Roughened respiration in right axilla. Bronchial respiration } \\
\text { at right apex posterior and over upper right back. Roughened respi- } \\
\text { ration over lower right back. Few clicks heard over upper right } \\
\text { back. loughened respiration over left back. Voice sounds near be- } \\
\text { low left clavicle. Vocal fremitus increased at left front. }\end{array}$ & $\begin{array}{l}\text { March } 6 . \\
109 \text { pounds. } \\
\text { (Has weighed 1121 } \\
\text { pounds.) } \\
\text { ऊ vi-viii. } \\
\text { Tubercle b a c illi } \\
\text { present. } \\
\text { 11.45 A.M. } \\
\text { Pulse 84. } \\
\text { Temp. } 100^{\circ} . \\
\text { Resp. } 22 .\end{array}$ & $\begin{array}{l}\text { May } 29 . \\
98 \text { pounds. } \\
3 \text { iii-iv. } \\
\text { Tubercle b a c illi } \\
\text { present. } \\
12.30 \text { P. M. } \\
\text { Pulse } 88 . \\
\text { Temp. 100. } \\
\text { Resp. } 26 .\end{array}$ & $\begin{array}{l}22 \\
48 \text { drops } \\
6 \text { drops. }\end{array}$ \\
\hline $\begin{array}{l}\text { IV. } \\
\text { Femaje. } \\
\text { 23 years. } \\
\text { Single. } \\
\text { Seamstress. } \\
\text { Family history not } \\
\text { obtained. }\end{array}$ & $\begin{array}{l}\text { No cough. No expectoration. No loss of weight. Menses regular. } \\
\text { Appetite fair. Loss of strength. } \\
\text { No dulness. Sibilant râles over left front. Roughened respiration } \\
\text { all over right back. Few fine râles at right apex posterior. }\end{array}$ & $\begin{array}{l}\text { March } 6 . \\
100 \text { pounds. } \\
\text { No sputum. } \\
12 \text { M. } \\
\text { Pulse } 80 . \\
\text { Temp. } 92.2^{\circ} \text {. } \\
\text { Resp. } 24 .\end{array}$ & $\begin{array}{l}\text { April } 12 . \\
99 \text { pounds. } \\
\text { No sputum. } \\
12.40 \text { P. M. } \\
\text { Pulse } 92 . \\
\text { Temp. } 98.6^{\circ} \text {. } \\
\text { Resp. } 23 .\end{array}$ & $\begin{array}{l}11 \\
11 \frac{1}{2} \text { drops } \\
2 \text { drops. }\end{array}$ \\
\hline $\begin{array}{l}\text { V. } \\
\text { Female. } \\
\text { 20 years. } \\
\text { Single. } \\
\text { Tobacco stripper. } \\
\text { F a ther and one } \\
\text { brother died of } \\
\text { phthisis. }\end{array}$ & $\begin{array}{l}\text { Cough seven months. No night sweats. No hæmoptysis. No loss } \\
\text { of weight. Menses regular. Feels fairly strong. No dyspnœa. Ap- } \\
\text { petite fair. } \\
\text { Dulness at right apex, anterior and posterior, and below right } \\
\text { clavicle. Cracked hot sound over 2d right interspace (near sternum). } \\
\text { Bronchial respiration over right front and back, and left back. } \\
\text { Roughened respiration over left front. Few medium râles at right } \\
\text { apex anterior, over upper right front, in right axilla, and between } \\
\text { right scapula and spine. Suspicion of râles between left scapula and } \\
\text { spine. Voice very near below right clavicle. }\end{array}$ & $\begin{array}{l}\text { March } 11 . \\
119 \frac{1}{2} \text { pounds. } \\
\text { Tubercle } \mathrm{i} \text { a c i } 11 \mathrm{i} \\
\text { present. } \\
\text { 12.45 P. M. } \\
\text { Pulse } 108 . \\
\text { Temp. } 100^{\circ} . \\
\text { Resp. } 24 .\end{array}$ & $\begin{array}{l}\text { April 22. } \\
113 \text { pounds. } \\
\text { Tubercle b a cilli } \\
\text { present. } \\
12 \text { s. } \\
\text { Pulse } 96 . \\
\text { Temp. 99. } \\
\text { Resp. 24. }\end{array}$ & $\begin{array}{l}17 \\
23 \text { drops. } \\
4 \text { drops. }\end{array}$ \\
\hline $\begin{array}{c}\text { VI. } \\
\text { Female. } \\
29 \text { years. } \\
\text { Married. } \\
\text { (3 children, young- } \\
\text { est } 3 \text { months old.) } \\
\text { Home-work. } \\
\text { Family history neg- } \\
\text { ative. }\end{array}$ & $\begin{array}{l}\text { Cough } 7 \text { months. No hæmoptysis. Occasional sweats. Has lost } \\
\text { flesh. Does not feel weak. Menses regular. No dyspncea. Appe- } \\
\text { tite poor. } \\
\text { Hectic flush. Considerable emaciation. Right clavicle more prom- } \\
\text { inent than left. Relative dulness not marked. Broncho-vesicular } \\
\text { respiration at left and right apex anterior. Slightly roughened res- } \\
\text { piration at left apex posterior. Fine moist râles at each apex ante- } \\
\text { rior. Sibilant râles at left apex anterior, in right axilla and over } \\
\text { right back. Moist râles between right scapula and spine. Pleuritic } \\
\text { stitch and few pleural râles in right anterior axillary line. }\end{array}$ & $\begin{array}{l}\text { March 14. } \\
\text { 92 pounds. } \\
\text { 3 iii-iv. } \\
\text { Tubercle b a c illi } \\
\text { present. } \\
\text { 1.30 P M. } \\
\text { Pulse 100. } \\
\text { Temp. 100. } \\
\text { Resp. 26. }\end{array}$ & $\begin{array}{l}\text { May } 25 . \\
88 \frac{1}{2} \text { pounds. } \\
3 \text { iss-ii. } \\
\text { Tubercle b a c i } 11 \mathrm{i} \\
\text { present. } \\
\text { 12.30 P. M. } \\
\text { Pulse 76. } \\
\text { Temp. } 98.4^{\circ} \\
\text { Resp. } 25 .\end{array}$ & $\begin{array}{l}17 \\
29 \text { drops. } \\
5 \text { drops. }\end{array}$ \\
\hline $\begin{array}{l}\text { VII. } \\
\text { Male. } \\
43 \text { years. } \\
\text { Single. } \\
\text { Baker. } \\
\text { Family history neg- } \\
\text { ative. }\end{array}$ & $\begin{array}{l}\text { Cough } 3 \text { months. No expectoration. No hromoptysis. Night } \\
\text { sweats for the last two months. Has lost fesh. Appetite poor. } \\
\text { Right clavicle more prominent than left. Relative dulness not } \\
\text { marked. Broncho-vesicular respiration at right apex and over right } \\
\text { front. Rather harsh respiration over right front near sternum. Bron- } \\
\text { cho-vesicular respiration in left axilla. Increased vesicular respiration } \\
\text { in right axilla. Roughened respiration in right anterior axillary } \\
\text { line. Broncho-vesicular respiration over upper half left back. Sibi- } \\
\text { lant râles at left apex and over left front. Medium and fine râles at } \\
\text { left apex anterior and over left front. Medium râles in left axilla. } \\
\text { Sibilant râles over upper half left back. Medium and fine râles over } \\
\text { upper half left back. }\end{array}$ & $\begin{array}{l}\text { March } 20 . \\
115 \text { pounds. } \\
\text { No sputum. } \\
\text { 2.30 P. M. } \\
\text { Pulse } 80 . \\
\text { Temp. } 97.4^{\circ} \text {. } \\
\text { Resp. 24. }\end{array}$ & $\begin{array}{l}\text { June } 8 . \\
115 \text { pounds. } \\
\text { No sputum. } \\
12.30 \text { P. M. } \\
\text { Pulse } 88 . \\
\text { Temp. 99.8 } \\
\text { Resp. } 22 .\end{array}$ & $\begin{array}{l}25 \\
79 \text { drops. } \\
6 \text { drops. }\end{array}$ \\
\hline $\begin{array}{l}\text { VIII. } \\
\text { Male. } \\
\text { 34 years. } \\
\text { Single. } \\
\text { Laborer. } \\
\text { milv historv n }\end{array}$ & $\begin{array}{l}\text { Cough } 2 \text { years. Pneumonia in November, 1892. Cough now mod- } \\
\text { erate. Expectoration mostly mucus. No night sweats (formerly had } \\
\text { them). Has lost flesh (gaining now). Appetite fair. } \\
\text { Relative dulness not especially marked. Roughened respiration } \\
\text { over right front and in each axilla. Harsh respiration at right apex } \\
\text { nosterior. Roughened respiration over right back and over lower }\end{array}$ & $\begin{array}{l}\text { April 17. } \\
\text { 166 pounds. } \\
\text { (Former weight } 195 \\
\text { pounds.) } \\
\text { Tntri. }\end{array}$ & $\begin{array}{l}\text { June } 23 . \\
169 \text { pounds. } \\
\text { 3 ii-iii. } \\
\text { Tubercle ba c } 111 \mathrm{i} \\
\text { present. }\end{array}$ & $\begin{array}{l}15 \\
30 \text { drops. } \\
3 \text { drops. }\end{array}$ \\
\hline
\end{tabular}


CUTANEOUS INJECtions OF GOLd AND MANGaNese (Dr. Boardman).

\begin{tabular}{|c|c|c|c|c|}
\hline nt. & $\begin{array}{l}\text { First Treatment. } \\
\text { Weight. } \\
\text { Sputum. }\end{array}$ & $\begin{array}{l}\text { Last Treatment. } \\
\text { Weight. } \\
\text { Sputum. }\end{array}$ & $\begin{array}{l}\text { Subcus. } \\
\text { Total Amount. } \\
\text { Largest Dose. }\end{array}$ & Physical Examination at the End of Treatment. \\
\hline $\begin{array}{l}\text { Loss of flesh } \\
\text { reats. Menses } \\
\text { le more prom- } \\
\text { hened nearly } \\
\text { Iedium moist }\end{array}$ & $\begin{array}{l}\text { Feb. 6, } 1893 . \\
111 \text { pounds. } \\
3 \text { fiss-iii. } \\
\text { No tubercle bacilli } \\
\text { found. } \\
11 \text { A. M. } \\
\text { Pulse 76. } \\
\text { Temp. 99. } \\
\text { Resp. 31. }\end{array}$ & $\begin{array}{l}\text { June } 13,1893 . \\
108 \frac{1}{2} \text { pounds. } \\
\text { (Did weigh } 117 \text { lbs. } \\
\text { after } 34 \text { subcus., } \\
\text { May 6th.) } \\
\text { 3 i-iss. } \\
\text { Tubercle bac illi } \\
\text { fairly numerous. } \\
\text { 12.30 P. M. } \\
\text { Pulse } 68 . \\
\text { Temp. } 99.3^{\circ} . \\
\text { Resp. } 35 .\end{array}$ & $\begin{array}{l}50 \\
112 \text { drops. } \\
6 \text { drops. }\end{array}$ & 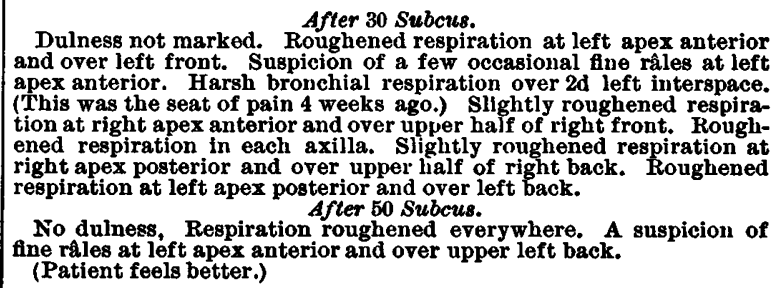 \\
\hline $\begin{array}{l}\text { th. Hoarse } \\
\text { optysis. No } \\
\text { lay, scanty). } \\
\text { over right } \\
\text { cord. }\end{array}$ & $\begin{array}{l}\text { Feb. 17. } \\
115 \text { pounds. } \\
\text { No sputum. } \\
\text { (Former weight 130 } \\
\text { pounds.) } \\
\text { 11 A. M. } \\
\text { Pulse 96. } \\
\text { Temp.98.8\%. } \\
\text { Resp. 21. }\end{array}$ & $\begin{array}{l}\text { April } 26 . \\
\text { 11ts pounds. } \\
\text { No sputum. } \\
12 \mathrm{M.} \\
\text { Pulse } 84 . \\
\text { Temp. } 99^{\circ} \text {. } \\
\text { Resp. } 21 .\end{array}$ & $\begin{array}{l}21 \\
29 \text { drops. } \\
5 \text { drops. }\end{array}$ & $\begin{array}{l}\text { No dulness. Fine moist râles at right apex anterior, under right } \\
\text { clavicle and over upper half of right chest anterior. Respiration } \\
\text { slightly roughened over lower half of right chest, in right axilla, over } \\
\text { left chest and in left axilla. Fine moist rales at left apex posterior, } \\
\text { between left scapula and spine, at right apex posterior and between } \\
\text { right scapula and spine. Left vocal cord ulcerated nearly its entire } \\
\text { length and considerably thickened. } \\
\text { (Patient feels better.) }\end{array}$ \\
\hline $\begin{array}{l}\text { Fairly strong. } \\
\text { left clavicle. } \\
\text { er half of left } \\
\text { oughened res- } \\
\text { leard at right } \\
\text { iâles in left } \\
\text { ial respiration } \\
\text { ighened respi- } \\
\text { rupper right } \\
\text { unds near be- } \\
\text { t. }\end{array}$ & $\begin{array}{l}\text { March } 6 . \\
109 \text { pounds. } \\
\text { (Has weighed 1121 } \\
\text { pounds.) } \\
\text { 3 vi-viii. } \\
\text { Tubercle b a c illi } \\
\text { present. } \\
\text { 11.45 A.M. } \\
\text { Pulse } 84 . \\
\text { Temp. } 100^{\circ} \text {. } \\
\text { Resp. 22. }\end{array}$ & 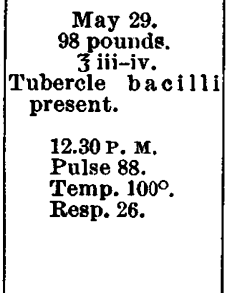 & $\begin{array}{l}22 \\
48 \text { drops. } \\
6 \text { drops. }\end{array}$ & $\begin{array}{l}\text { Relative dulness at left apex anterior and below left clavicle. } \\
\text { Cracked-pot sound under left clavicle. Bronchial respiration at left } \\
\text { apex anterior and all over left front. Medium and coarse rales at } \\
\text { left apex anterior and all over left front. Clicks heard at right apex } \\
\text { anterior and under right clavicle. Roughened respiration over upper } \\
\text { half of right front. Medium and tine râles in left axilla. Sibilalit } \\
\text { râles in right axilia. Respiration rough and harsh at right apex } \\
\text { posterior and above right scapula. Clicks heard at right apex pos- } \\
\text { terior and between right scapnla and spine. Fine râles at left apex } \\
\text { posterior. Fine and medium râles over left back Voice very near } \\
\text { under left clavicle and between right scapula and spine. Vocal fre- } \\
\text { mitus increased at left front. } \\
\text { (Patient feels about the same as before treatment.) }\end{array}$ \\
\hline $\begin{array}{l}\text { ses regular. } \\
\text { respiration }\end{array}$ & $\begin{array}{l}\text { March } 6 . \\
100 \text { pounds. } \\
\text { No sputum. } \\
12 \text { M. } \\
\text { Pulse } 80 \text {. } \\
\text { Temp. } 92.2 \% \text {. } \\
\text { Resp. } 24 .\end{array}$ & $\begin{array}{l}\text { April } 12 . \\
991 \text { pounds. } \\
\text { No sputum. } \\
12.40 \text { P. M. } \\
\text { Pulse } 92 . \\
\text { Temp. } 98.6^{\circ} \text {. } \\
\text { Resp. } 23 .\end{array}$ & $\begin{array}{l}11 \\
11 \frac{1}{2} \text { drops. } \\
2 \text { drops. }\end{array}$ & $\begin{array}{l}\text { No relative dulness. Respiration roughened everywhere. Sibilant } \\
\text { râles at npper left front. Voice near between right scapula and } \\
\text { spine. } \\
\text { (Patient feels about the same as before treatment.) }\end{array}$ \\
\hline $\begin{array}{l}\text { is. No loss } \\
\text { pnca. Ap- } \\
\text { below right } \\
\text { r sternum). } \\
\text { left back. } \\
\text { les at right } \\
\text { nd between } \\
\text { capula and }\end{array}$ & $\begin{array}{l}\text { March } 11 . \\
1191 \text { pounds. } \\
3 \mathrm{i} . \\
\text { Tubercle b a c i } 11 \mathrm{i} \\
\text { present. } \\
12.45 \text { P. } \mathrm{sr} . \\
\text { Pulse } 108 . \\
\text { Temp. 100. } \\
\text { Resp. 24. }\end{array}$ & $\begin{array}{l}\text { April 22. } \\
113 \text { pounds. } \\
\text { 3 i. } \\
\text { Tubercle b a cilli } \\
\text { present. } \\
\text { 12 3r. } \\
\text { Pulse 96. } \\
\text { Temp. 99. } \\
\text { Resp. 24. }\end{array}$ & $\begin{array}{l}17 \\
23 \text { drops. } \\
4 \text { drops. }\end{array}$ & $\begin{array}{l}\text { Dulness at right apex anterior below right clavicle, at right apex } \\
\text { posterior and over upper half of right back. Cracked-pot sound over } \\
\text { 2d right interspace. Bronchial respiration over upper half of right } \\
\text { and left chest, anterior and posterior. Broncho-vesieular respiration } \\
\text { in right axilla. Roughened respiration in left axilla and over lower } \\
\text { right and left back. Respiration rather amphoris over } 2 \mathrm{~d} \text { right inter- } \\
\text { space. Medium râles at right apex anterior, over upper right front } \\
\text { and in right axilla. Numerous clicks at right apex posterior and } \\
\text { over upper right back. Suspicion of fine râles at left apex posterior. } \\
\text { Occasional fine râles at left apex anterior. Medium râles below left } \\
\text { clavicle. Voice very near at upper right chest, anterior and pos- } \\
\text { terior. } \\
\text { (Patient feels better.) }\end{array}$ \\
\hline $\begin{array}{l}\text { Has lost } \\
\text { cea. Appe- } \\
\text { more prom- } \\
\text { lo-vesicular } \\
\text { ghened res- } \\
\text { apex ante- } \\
\text { la and over } \\
\text { Pleuritic }\end{array}$ & $\begin{array}{l}\text { March 14. } \\
92 \text { pounds. } \\
3 \text { iii-iv. } \\
\text { Tubercle b ac ill i } \\
\text { present. } \\
\text { 1.30 P M. } \\
\text { Pulse 100. } \\
\text { Temp. 100. } \\
\text { Resp. 26. }\end{array}$ & 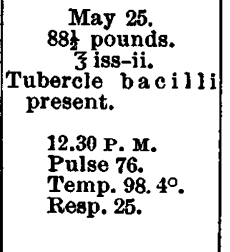 & $\begin{array}{c}17 \\
29 \text { drops. } \\
5 \text { drops. }\end{array}$ & $\begin{array}{l}\text { Relative dulness not marked. Respiration slightly roughened be- } \\
\text { low left clavicle, broncho-vesicular at right apex a.iterior and over } \\
\text { upper right front, bronchial at right apex posterior, roughened above } \\
\text { right scapula, slightly roughened at left apex posterior and over upper } \\
\text { left back. Clicks heard at left apex. Few fine moist râles at left } \\
\text { apex anterior. Numerous fine râles at right apex anterior. Fine } \\
\text { râles under right clavicle Suspicion of fine râles at right apex } \\
\text { posterior. Fine râles between right scapula and spine. Voice nearer } \\
\text { over right upper front and back. Vocal fremitus increased over right } \\
\text { upper front and back. } \\
\text { (Patient feels better.) }\end{array}$ \\
\hline $\begin{array}{l}\text { tysis. Night } \\
\text { ite poor. } \\
\text { dulness not } \\
\text { ind over right } \\
\text { rnum. Bron- } \\
\text { ar respiration } \\
\text { rior axillary } \\
\text { t back. Sibi- } \\
\text { fine râles at } \\
\text { in left axilla. } \\
\text { ine râles over }\end{array}$ & $\begin{array}{l}\text { March } 20 . \\
115 \text { pounds. } \\
\text { No sputum. } \\
230 \text { P. M. } \\
\text { Pulse } 80 . \\
\text { Temp. 97.40. } \\
\text { Resp. 24. }\end{array}$ & $\begin{array}{l}\text { June 8. } \\
115 \text { pounds. } \\
\text { No sputum. } \\
\text { 12.30 P. M. } \\
\text { Pulse 88. } \\
\text { Temp. 99.8 } \\
\text { Resp. 22. }\end{array}$ & $\begin{array}{l}25 \\
79 \text { drops. } \\
6 \text { drops. }\end{array}$ & $\begin{array}{l}\text { Coughs less than before. No expectoration. Still sweats a little at } \\
\text { night. Appetite poor. No relative dulness. Slightly roughened } \\
\text { respiration over middle of left front and at lower part of right axilla. } \\
\text { Fine moist râles at left apex anterior and under left clavicle. Sibilant } \\
\text { râles over left front. Right apex and front chiefly negative. Fine } \\
\text { moist râles in left axilla. Signs chiefly negative in right axilla and } \\
\text { over right back. (Very fine crepitation at inferior angle of right } \\
\text { scapula.) Fine moist râles at left apex posterior. Occasional sibilant } \\
\text { rales over left back. } \\
\text { (Patient feels better.) }\end{array}$ \\
\hline $\begin{array}{l}\text { now mod- } \\
\text { rmerly had } \\
\text { respiration } \\
\text { right apex } \\
\text { over lower }\end{array}$ & $\mid \begin{array}{c}\text { April 17. } \\
\text { 166 pounds. } \\
\text { (Former weight 195 } \\
\text { pounds.) } \\
\text { 3 v-vi. } \\
\text { Tnherele hac il } 1 \text { i }\end{array}$ & $\mid \begin{array}{c}\text { June } 23 . \\
169 \text { pounds. } \\
\text { 3 ii-iii. } \\
\text { Tubercle b a c ill i } \\
\text { present. }\end{array}$ & $\begin{array}{c}15 \\
30 \text { drops. } \\
3 \text { drops. }\end{array}$ & $\begin{array}{l}\text { Relative dulness not marked. Broncho-vesicular respiration at left } \\
\text { apex anterior. Slightly roughened respiration over left front. } \\
\text { Broncho-vesicular respiration at right apex anterior. Roughened } \\
\text { respiration over right front and in each axilla. Broncho-vesicular } \\
\text { respiration at right apex posterior. Ronghened respiration over right } \\
\text { hack. Sliohtlv ronghened resniration at. lower left hack. Fine moist. }\end{array}$ \\
\hline
\end{tabular}


Cough seven months. No night sweats. No hæmoptysis, No loss of weight. Menses regular. Feels fairly strong. No dyspncea. Ap-

pétite fair. clavicle. Cracked hot sound over $2 d$ right interspace (near sternum) Bronchial respiration over right frout and back aud left back. Roughened respiration over left front. Few medium râles at right apex anterior, over upper right front, in right axilla, and between apex anterior, over upper right front, in right axilla, and between spine. Voice very near below right clavicle.

VI.

Female.

29 years.

Married.

( 3 children, young

Home-work.

Family history negative.

\section{VII. \\ Male. \\ 43 years. \\ Single. \\ Family history neg- ative.}

Cough 7 months. No hæmoptysis. Occasional sweats. Has lost lesh. Does not feel weak. Menses regular. No dyspncea. Appetite poor

Hectic flush. Considerable emaciation. Right clavicle more prominent than left. Relative dulness not marked. Broncho-vesicular respiration at left and right apex anterior. Slightly roughened res. piration at left apex posterior. Fine moist râles at each apex anterior. Sibilant râles at left apex anterior, in right axilla and ove right back. Moist râles between right scapula and spine. Pleuritic stitch and few pleural râles in right anterior axillary line.

Cough 3 months. No expectoration. No hæmoptysis. Night sweats for the last two months. Has lost flesh. Appetite poor.

Right clavicle more prominent than left. Relative dulness not marked. Broncho-vesicular respiration at right apex and over right front. Rather harsh respiration over right front near sternum. Broncho-vesicular respiration in left axilla. Increased vesicular respiration in right axilla. Roughened respiration in right anterior axillary line. Broncho-vesicular respiration over upper half left back. Sibilant râles at left apex and over left front. Medium and fine râles at left apex anterior and over left front. Medium râles in left axilla. Sibilant râles over upper half left back. Medium and fine râles over upper half left back.

VIII.
Male.
34 years.
Single.
Laborer.
Family history neg-
ative.

Cough 2 years. Pneumonia in November, 1892. Cough now moderate. Expectoration mostly mucus. No night sweats (formerly had hem). Has lost flesh (gaining now). Appetite fair.

Relative dulness not especially marked. Roughened respiration over right front and in each axilla. Harsh respiration at right apex posterior. Roughened respiration over right back and over lower
half left back. Fine moist râles at left apex anterior and below left clavicle. Occasional fine râles at right apex anterior.

Larynx. Thickening of interarytenoid fold. Enlarged and immovable right arytenoid. No movement of right vocal cord.

Cough 4 months. Expectoration scanty, muco-purulent. HæmopIX.

35 years.

Married.

Laborer.

Family history neg. ative. Cis 4 years ago. Has lost fiesh. A ppetite poor. Relative dulness not especially marked. Roughened respiration in right axilla. Bronright apex posterior picion of fine râles at left apex anterior. Medium râles at right apex anterior. Suspicion of fine râles at left apex posterior. Sibilant râles over left and right backs. Fine râles at right apex posterior.

Cough one year. Expectoration muco-purulent, No hæmoptysis. Male.

Male.

46 years.

Furniture mover. Family history negative. and over left front, at right apex posterior and over right and left cks. Broncho-vesicular respiration at right apex anterior and over ght front, and in each axilla. Fine, moist râles at left apex anteMedium, moist râles over left front. Fine and medium râles at right apex anterior, over right front, at right apex posterior, and axilla and over upper balf left back. Medium râles in right axilla. $19 \frac{1}{2}$ pounds.

April 22.

ubercle $\mathrm{b}$. 113 pounds.

$12.45 \mathrm{P} . \mathrm{M}$.

Pulse 108 .

Temp. $100^{\circ}$

$12 \mathrm{M}$.

$12 \mathrm{Mr}$.

Pulse 96.

Temp. $99^{\circ}$.
Resp. 24.

Resp. 24.

Tu

March 14

92 pounds.

3 iii-iv.

May 25.

$88 \frac{1}{2}$ pounds

17 .

pubercle b
present.

$1.30 \mathrm{P}$ M.

Pulse 100.

Temp. $100^{\circ}$

Resp. 26.

Tubercle b

29 drops. present.

5 drops.

\section{March 20. \\ 115 pounds.}

No sputum.

12.30 P.

Pulse 76.

Temp. 98. $4^{\circ}$.

Resp. 25.

2.30 P. M.

Pulse 80.

Temp. 97.

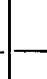

June 8

115 pounds.

No sputum.

25

$12.30 \mathrm{P} . \mathrm{M}$.

Pulse 88.

Resp. 22.

79 drops.

6 drops.

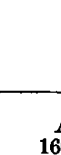

April 17. Former weight 195 pounds.)

Tubercle bi.

present.

1 P. M.

Puise 76.

Temp. 97

Resp. 24.

April 18.

121 pounds.

Former wejght 146

pounds.)

No tubercle bacill

found.

1.30 P. M.

Pulse 76.

Resp. 34 .

April 19.

117 pounds.

117 pounds.
3 iss.
Tubercle ba cill i
Tubercle baci $11 \mathrm{i}$

present.

June 23

169 pounds.

Tubercle bacilli

present.

11 A. M.

Tulse 64.

Resp. 24.

1.20 P. M.

Pulse 88.

Temp 99.
Resp. 28.

SUMMARY OF ALL CASES TREATED AND RESU.

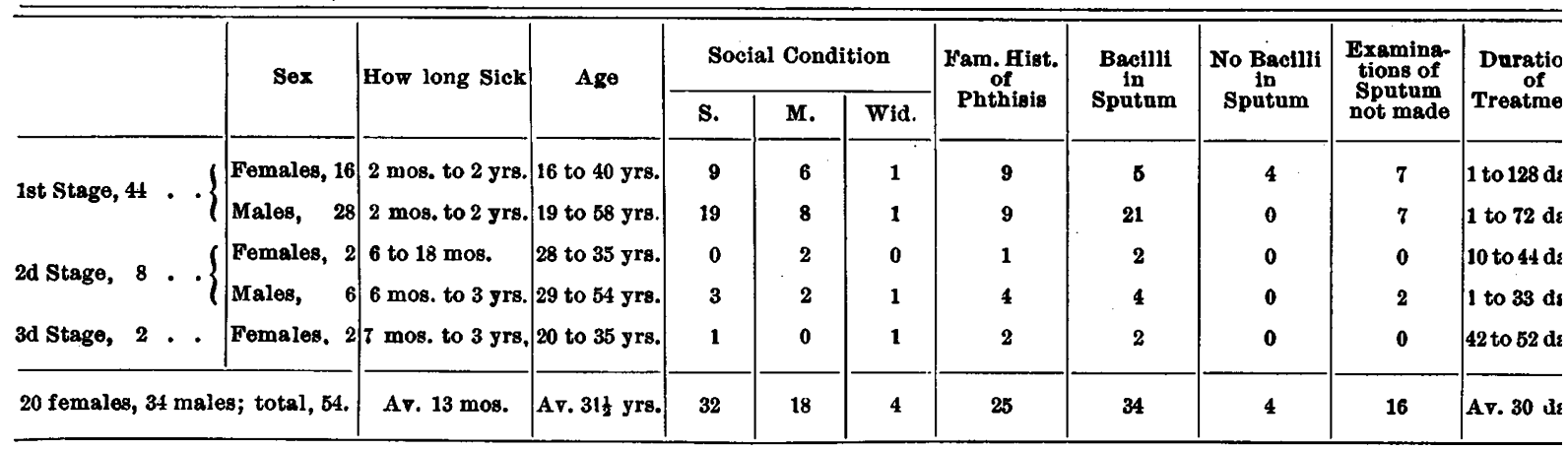




\begin{tabular}{|c|c|c|c|c|}
\hline $\begin{array}{l}\text { sis. No loss } \\
\text { spncea. Ap- } \\
\text { below right } \\
\text { ar sternum). } \\
\text { left back. } \\
\text { les at right } \\
\text { nd between } \\
\text { scapula and }\end{array}$ & $\begin{array}{l}\text { March 11. } \\
119+\text { pounds. } \\
\text { Z i. } \\
\text { Tubercle b a c i } 11 \mathrm{i} \\
\text { present. } \\
12.45 \text { P. M. } \\
\text { Pulse } 108 . \\
\text { Temp. 100. } \\
\text { Resp. 24. }\end{array}$ & $\begin{array}{l}\text { April 22. } \\
113 \text { pounds. } \\
\text { 3 i. } \\
\text { Tubercle b a ci } 11 \text { i } \\
\text { present. } \\
12 \text { Mr. } \\
\text { Pulse 96. } \\
\text { Temp. 99. } \\
\text { Resp. 24. }\end{array}$ & $\begin{array}{c}17 \\
23 \text { drops. } \\
4 \text { drops. }\end{array}$ & $\begin{array}{l}\text { Dulness at right apex anterior below right clavicle, at right apex } \\
\text { posterior and over upper half of right back. Cracked-pot sound over } \\
\text { 2d right interspace Bronchial respiration over upper half of right } \\
\text { and left chest, anterior and posterior. Broncho-vesicnlar respiration } \\
\text { in right axilla. Roughened respiration in left axilla and over lower } \\
\text { right and left back. Respiration rather amphoric over } 2 d \text { right inter- } \\
\text { space. Medium râles at right apex anterior, over upper right front } \\
\text { and in right axilla. Numerous clicks at right apex posterior and } \\
\text { over upper right back. Suspicion of fine râles at left apex posterior. } \\
\text { Occasional fine râles at left apex anterior. Medium râles below left } \\
\text { clavicle. Voice very near at upper right chest, anterior and pos- } \\
\text { terior. } \\
\text { (Patient feels better.) }\end{array}$ \\
\hline $\begin{array}{l}\text { Has lost } \\
\text { nœea. Appe- } \\
\text { more prom- } \\
\text { ho-vesicular } \\
\text { ighened res- } \\
\text { apex ante- } \\
\text { illa and over } \\
e_{\text {. Pleuritic }} \\
\text { le. }\end{array}$ & \begin{tabular}{|} 
March 14. \\
92 pounds. \\
3 iii-iv. \\
Tubercle b a c ill i \\
present. \\
\\
1.30 P M. \\
Pulse 100. \\
Temp. 100. \\
Resp. 26.
\end{tabular} & \begin{tabular}{|c|} 
May 25. \\
88t pounds. \\
3 iss-ii. \\
Tubercle b a c i $11 \mathrm{i}$ \\
present. \\
\\
12.30 P. M. \\
Pulse 76. \\
Temp. 98. $4^{\circ}$. \\
Resp. 25.
\end{tabular} & $\begin{array}{l}17 \\
29 \text { drops. } \\
5 \text { drops. }\end{array}$ & $\begin{array}{l}\text { Relative dulness not marked. Respiration slightly roughened be- } \\
\text { low left clavicle, broncho-vesicular at right apex anterior and over } \\
\text { upper right front, bronchial at right apex posterior, roughened above } \\
\text { right scapula, slightly roughened at left apex posterior ald over upper } \\
\text { left back. Clicks heard at left apex. Few fine moist râles at left } \\
\text { apex anterior. Numerous fine râles at right apex anterior. Fine } \\
\text { râles under right clavicle Suspicion of finte râles at right apex } \\
\text { posterior. Fine râles between right scapula and spine. Voice nearer } \\
\text { over right upper front and back. Vocal fremitus increased over right } \\
\text { upper front and back. } \\
\text { (Patient feels better.) }\end{array}$ \\
\hline $\begin{array}{l}\text { tysis. Night } \\
\text { ite poor. } \\
\text { dulness not } \\
\text { ind over right } \\
\text { rnum. Bron- } \\
\text { ar respiration } \\
\text { rior axillary } \\
\text { t back. Sibi- } \\
\text { ine râles at } \\
\text { in left axilla. } \\
\text { ine râles over }\end{array}$ & $\begin{array}{l}\text { March } 20 . \\
115 \text { pounds. } \\
\text { No sputum. } \\
2.30 \text { P. M. } \\
\text { Pulse } 80 . \\
\text { Temp. } 97.4^{\circ} \\
\text { Resp. } 24 \text {. }\end{array}$ & $\begin{array}{l}\text { June } 8 . \\
115 \text { pounds. } \\
\text { No sputum. } \\
\text { 12.30 P. M. } \\
\text { Pulse } 88 . \\
\text { Temp. } 99.8^{\circ} \\
\text { Resp. } 22 .\end{array}$ & $\begin{array}{c}25 \\
79 \text { drops. } \\
6 \text { drops. }\end{array}$ & $\begin{array}{l}\text { Coughs less than before. No expectoration. Still sweats a little at } \\
\text { night. Appetite poor. No relative dulness. Slightly roughened } \\
\text { respiration over middle of left front and at lower part of right axilla. } \\
\text { Fine moist râles at left apex anterior and under left clavicle. Sibilant } \\
\text { râles over left front. Right apex and front chiefly negative. Fine } \\
\text { moist râles in left axilla. Signs chiefy negative in right axilla and } \\
\text { over right back. (Very fine crepitation at inferior angle of right } \\
\text { scapula.) Fine moist râles at left apex posterior. Occasional sibilant } \\
\text { rales over left back. } \\
\text { (Patient feels better.) }\end{array}$ \\
\hline $\begin{array}{l}\text { gh now mod- } \\
\text { formerly had } \\
\text { d respiration } \\
\text { at right apex } \\
\text { d over lower } \\
\text { ind below left } \\
\text { rged and im- } \\
\text { cord. }\end{array}$ & $\begin{array}{l}\text { April 17. } \\
\text { 166 pounds. } \\
\text { (Former weight } 195 \\
\text { pounds.) } \\
\text { 3 v-vi. } \\
\text { Tubercle b a c illi } \\
\text { present. } \\
\text { 1 P. M. } \\
\text { Puise 76. } \\
\text { Temp. 97. } \\
\text { Resp. } 24 .\end{array}$ & $\begin{array}{l}\text { June } 23 . \\
169 \text { pounds. } \\
\text { 3 ii-iii. } \\
\text { Tubercle ba cill i } \\
\text { present. } \\
11 \text { A. M. } \\
\text { Pulse 64. } \\
\text { Temp. 96. } \\
\text { Resp. 24. }\end{array}$ & $\begin{array}{c}15 \\
30 \text { drops. } \\
3 \text { drops. }\end{array}$ & $\begin{array}{l}\text { Relative dulness not marked. Broncho-vesicular respiration at left } \\
\text { apex anterior. Slightly roughened respiration over left front. } \\
\text { Broncho-vesicular respiration at right apex anterior. Roughened } \\
\text { respiration over right front and in each axila. Broncho-vesicular } \\
\text { respiration at right apex posterior. Roughened respiration over right } \\
\text { back. Slightly roughened respiration at lower left back. Fine moist } \\
\text { rales at left apex anterior, under left clavicle and at right apex an- } \\
\text { terior. Few fine moist râles above left scapula. Larynx about the } \\
\text { same as before. } \\
\text { (Patient feels better.) }\end{array}$ \\
\hline $\begin{array}{l}\text { ent. Hæmop- } \\
\text { ative dulness } \\
\text { axilla. Bron- } \\
\text { back, and at } \\
\text { t back. Sus- } \\
\text { at right apex } \\
\text { ior. Sibilant } \\
\text { posterior. }\end{array}$ & $\begin{array}{l}\text { April } 18 . \\
\text { 121, pounds. } \\
\text { (Former weight } 146 \\
\text { pounds.) } \\
\text { ऊ ii. } \\
\text { No tubercle bacilli } \\
\text { found. } \\
\\
1.30 \text { P. M. } \\
\text { Pulse } 76 . \\
\text { Temp. } 98^{\circ} \text {. } \\
\text { Resp. } 34 .\end{array}$ & $\begin{array}{l}\text { June } 24 . \\
116 \frac{1}{2} \text { pounds. } \\
3 \text { ii. } \\
\text { S m a } 11 \text { number of } \\
\text { tubercle ba cilli } \\
\text { present. } \\
1 \text { P. M. } \\
\text { Puise 84. } \\
\text { Temp. 98.7. } \\
\text { Resp. } 30 \text {. }\end{array}$ & $\begin{array}{c}21 \\
67 \text { drops. } \\
6 \text { drops. }\end{array}$ & $\begin{array}{l}\text { No relative dulness. Broncho-vesicular respiration at left apex an- } \\
\text { terior. Slightly roughened respiration over upper half of left front. } \\
\text { Broncho-vesicular respiration at right apex anterior. Slightly rough- } \\
\text { ented respiration over right front and in each axilla. Broncho-vesicular } \\
\text { respiration at right apex posterior, over upper half of right back, at } \\
\text { left apex posterior and above lelt scapula. Occasional fine râles at } \\
\text { right apex anterior. Occasional fine and medium râles at right apex } \\
\text { posterior and between right scapula and spine. } \\
\text { (Patient feels better.) }\end{array}$ \\
\hline $\begin{array}{l}\text { hæmoptysis. } \\
\text { ppetite good. } \\
\text { at left apex } \\
\text { ight and left } \\
\text { rior and over } \\
\text { it apex ante- } \\
\text { dium râles at } \\
\text { osterior, and } \\
\text { per half left }\end{array}$ & $\begin{array}{l}\text { April 19. } \\
117 \frac{1}{2} \text { pounds. } \\
\text { ऊ iss. } \\
\text { Tubercle b a cilli } \\
\text { present. } \\
1.20 \text { P. M. } \\
\text { Pulse } 88^{\circ} \\
\text { Temp } 99^{\circ} \\
\text { Resp. } 28 .\end{array}$ & \begin{tabular}{|} 
June 16. \\
117 pounds. \\
$3 \mathrm{v}$. \\
Tubercle b a i ll i \\
present.
\end{tabular} & $\begin{array}{c}15 \\
45 \text { drops. } \\
4 \text { drops. }\end{array}$ & $\begin{array}{l}\text { Relative dulness not marked. Broncho-vesicular respiration at left } \\
\text { apex anterior. Bronchial respiration over upper half of left front. } \\
\text { Broncho-vesicular respiration at right apex ant. and over right front } \\
\text { and in each axilla. Bronchial respiration at right apex posterior, } \\
\text { over right back, at left apex posterior and over left back. Fine moist } \\
\text { râles at left apex anterior. Few sibilant râles at left apex anterior. } \\
\text { Fine moist râles over upper balf of left front. Medium moist râles at } \\
\text { right apex anterior, over right front and in right axilla. Fine moist } \\
\text { râles in left axilla. Medium moist râles at right apex posterior, over } \\
\text { right back and over upper half of left back. } \\
\text { (Patient feels " slightly better."). }\end{array}$ \\
\hline
\end{tabular}

IARY OF ALL CASES TREATED AND RESULTS.

\begin{tabular}{|c|c|c|c|c|c|c|c|c|c|c|c|c|}
\hline dition & \multirow{2}{*}{$\begin{array}{c}\text { Fam. Hist. } \\
\text { of } \\
\text { Phthigis }\end{array}$} & \multirow{2}{*}{$\begin{array}{c}\text { Bacilli } \\
\text { in } \\
\text { Sputam }\end{array}$} & \multirow{2}{*}{$\begin{array}{c}\text { No Bacilli } \\
\text { 1n } \\
\text { Sputum }\end{array}$} & \multirow{2}{*}{$\begin{array}{c}\text { Examina- } \\
\text { tions of } \\
\text { Sputum } \\
\text { not made }\end{array}$} & \multirow{2}{*}{$\begin{array}{c}\text { Duration } \\
\text { of } \\
\text { Treatment }\end{array}$} & \multirow{2}{*}{$\begin{array}{c}\text { Number } \\
\text { of } \\
\text { Injections }\end{array}$} & \multicolumn{3}{|c|}{ * Result on Examination } & \multicolumn{3}{|c|}{ Subjective Result } \\
\hline Wid. & & & & & & & Better & Not Imp. & Worse & Better & Not Imp. & Worse \\
\hline 1 & 9 & $\mathbf{5}$ & 4 & 7 & 1 to 128 days & 1 to 50 & 2 & $\mathbf{0}$ & $\mathbf{3}$ & 8 & 8 & $\mathbf{0}$ \\
\hline 1 & 9 & 21 & 0 & 7 & 1 to 72 days & 1 to 37 & 1 & 9 & 2 & 9 & 18 & $\mathbf{1}$ \\
\hline $\mathbf{0}$ & 1 & 2 & 0 & $\mathbf{0}$ & 10 to 44 days & 7 to 5 & 0 & $\mathbf{0}$ & $\mathbf{0}$ & $\mathbf{0}$ & 1 & 1 \\
\hline 1 & 4 & 4 & $\mathbf{0}$ & 2 & 1 to 33 days & 1 to 15 & $\mathbf{0}$ & $\mathbf{1}$ & $\mathbf{0}$ & $\mathbf{3}$ & 3 & $\mathbf{0}$ \\
\hline $\mathbf{1}$ & 2 & 2 & $\mathbf{0}$ & 0 & 42 to 52 days & 4 to 17 & $\mathbf{0}$ & 0 & 2 & 1 & 0 & 1 \\
\hline 4 & 25 & 34 & 4 & 16 & Av. 30 uays & Av. 9 & $\mathbf{3}$ & 10 & 7 & 21 & 30 & 3 \\
\hline
\end{tabular}

\section{P I. - Patients Treated with Gold and Manganege.}


SUMMARY OF ALL CASES TREATED AND RESU.

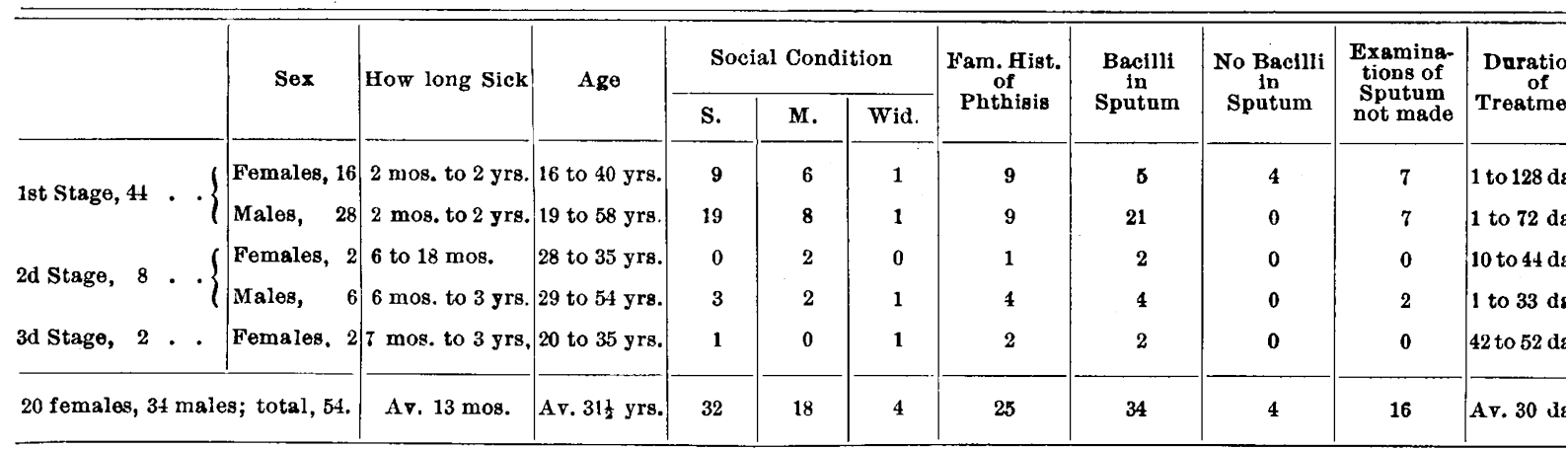

Group I. - Patients Treated with Gold and Mangan

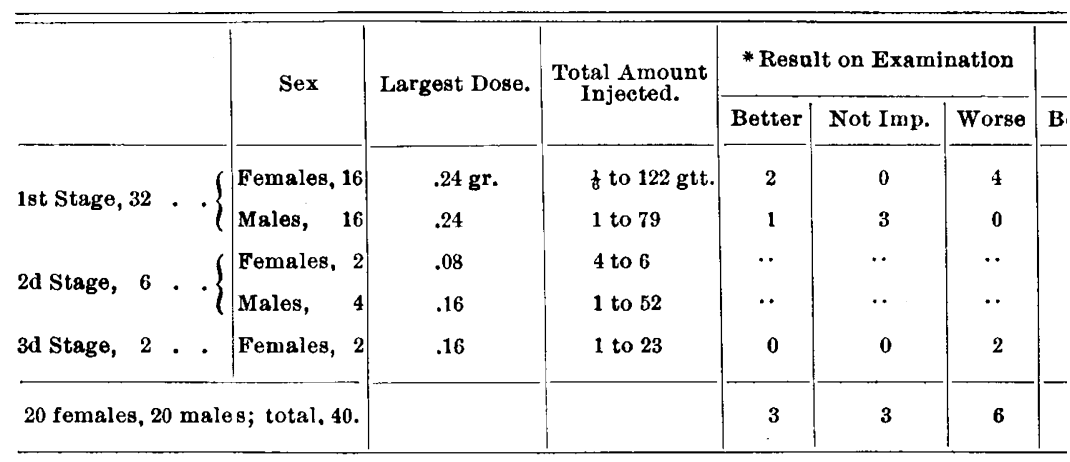

Group II. - Patients Treated with Hunter's Mod.

\begin{tabular}{|c|c|c|c|c|c|c|c|}
\hline 1st Stage, 12 & $\cdot\{$ & $\begin{array}{ll}\text { Females, } & 0 \\
\text { Males, } & 12\end{array}$ & $\begin{array}{l}. . \\
.125 \mathrm{gm} .\end{array}$ & $\begin{array}{c}. \\
.005 \text { to } 1.855 \mathrm{gm}\end{array}$ & $\begin{array}{l}\cdots \\
0\end{array}$ & $\begin{array}{l}\cdots \\
6\end{array}$ & $\cdot$ \\
\hline 2d Stage, 2 & $\cdot\{$ & $\begin{array}{ll}\text { Females, } & 0 \\
\text { Males, } & 2\end{array}$ & $\begin{array}{l}. \\
.075\end{array}$ & $\begin{array}{c}. \\
.025 \text { to } .075\end{array}$ & $\ddot{0}$ & $\begin{array}{l}\cdots \\
1\end{array}$ & $\begin{array}{l}\cdots \\
0\end{array}$ \\
\hline 3d Stage, 0 & . . & $\cdots \cdots \quad 0$ & .. & .. & .. & 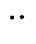 & .. \\
\hline Nofemales, & $4 \mathrm{mal}$ & es; total 14 & & & 0 & 7 & $\mathbf{1}$ \\
\hline
\end{tabular}

Group III (taken from Group I). - Patients Treated First with Gol and Later with Hunter's Mod. B.

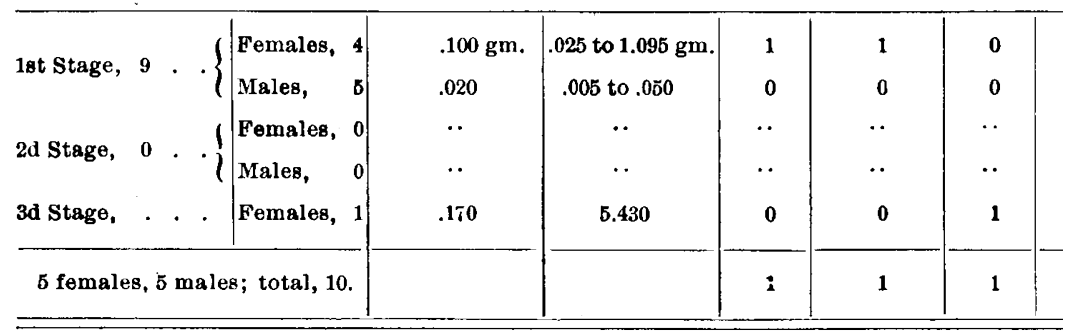

* The result on physical examination conld not be ascertained in every case, since some ps unexpectedly, before a final examination could be made. 


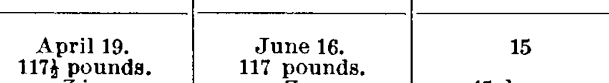

Relative dulness not marked. Broncho-vesicular respiration at left $117 t$ pounds. 117 pounds.

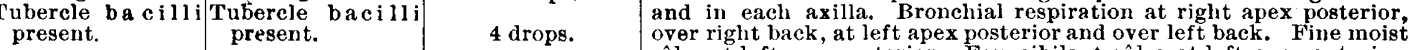
present. râles at left apex anterior. Few sibilant râles at left apex anterior. Fine moist râles over upper half of left front. Medium moist râles at right apex anterior, over right front and in right axilla. Fine moist Pulse 88. Temp $99^{\circ}$ râles in left axilla. Medium moist râles at right apex posterior, over right back and over upper half of left back.

AARY OF ALL CASES TREATED AND RESULTS.

\begin{tabular}{|c|c|c|c|c|c|c|c|c|c|c|c|c|}
\hline \multirow[t]{2}{*}{ dition } & \multirow{2}{*}{$\begin{array}{c}\text { Fam. Hist. } \\
\text { of } \\
\text { Phthisis }\end{array}$} & \multirow{2}{*}{$\begin{array}{c}\text { Bacilli } \\
\text { in } \\
\text { Sputam }\end{array}$} & \multirow{2}{*}{$\begin{array}{c}\text { No Bacilli } \\
\text { 1n } \\
\text { Sputum }\end{array}$} & \multirow{2}{*}{$\begin{array}{c}\text { Examina- } \\
\text { tions of } \\
\text { Sputum } \\
\text { not made }\end{array}$} & \multirow{2}{*}{$\begin{array}{c}\begin{array}{c}\text { Daration } \\
\text { of } \\
\text { Treatment }\end{array} \\
\end{array}$} & \multirow{2}{*}{$\begin{array}{c}\text { Number } \\
\text { of } \\
\text { Injections }\end{array}$} & \multicolumn{3}{|c|}{ * Rosult on Examination } & \multicolumn{3}{|c|}{ Subjective Result } \\
\hline & & & & & & & Better & Not Imp. & Worse & Better & Not Imp. & Worse \\
\hline 1 & 9 & 5 & 4 & 7 & 1 to 128 days & 1 to 50 & 2 & $\mathbf{0}$ & 3 & 8 & 8 & $\mathbf{0}$ \\
\hline 1 & 9 & 21 & 0 & 7 & 1 to 72 days & 1 to 37 & 1 & 9 & 2 & 9 & 18 & 1 \\
\hline 0 & 1 & 2 & 0 & 0 & 10 to 44 days & 7 to 5 & 0 & 0 & $\mathbf{0}$ & 0 & 1 & 1 \\
\hline 1 & 4 & 4 & 0 & 2 & 1 to 33 days & 1 to 15 & 0 & $\mathbf{1}$ & 0 & 3 & 3 & 0 \\
\hline 1 & 2 & 2 & o & 0 & 42 to $52 \mathrm{days}$ & 4 to 17 & $\mathbf{0}$ & 0 & 2 & 1 & 0 & 1 \\
\hline 4 & 25 & 34 & 4 & 16 & Av. 30 days & Av. 9 & 3 & 10 & 7 & $2 t$ & 30 & 3 \\
\hline
\end{tabular}

\section{P I. - Patients Treated with Gold and Manganese.}

\begin{tabular}{|c|c|c|c|c|c|c|c|}
\hline \multirow{2}{*}{ Largest Dose. } & \multirow{2}{*}{$\begin{array}{l}\text { Total Amount } \\
\text { Injected. }\end{array}$} & \multicolumn{3}{|c|}{ * Result on Examination } & \multicolumn{3}{|c|}{ Subjective Result } \\
\hline & & Better & Not Imp. & Worse & Better & Not Imp. & Worse \\
\hline $.24 \mathrm{gr}$. & f to $122 \mathrm{gtt}$. & 2 & 0 & 4 & 8 & 8 & 0 \\
\hline .24 & 1 to 79 & 1 & 3 & 0 & 6 & 10 & 0 \\
\hline .08 & 4 to 6 & * & . & . & 0 & 1 & 1 \\
\hline .16 & 1 to 52 & .. & . & . & 2 & 2 & 0 \\
\hline .16 & 1 to 23 & 0 & 0 & 2 & 1 & 0 & 1 \\
\hline & & 3 & 3 & 6 & 17 & 21 & 2 \\
\hline
\end{tabular}

dup II. - Patients Treated with Hunter's Mod. B.

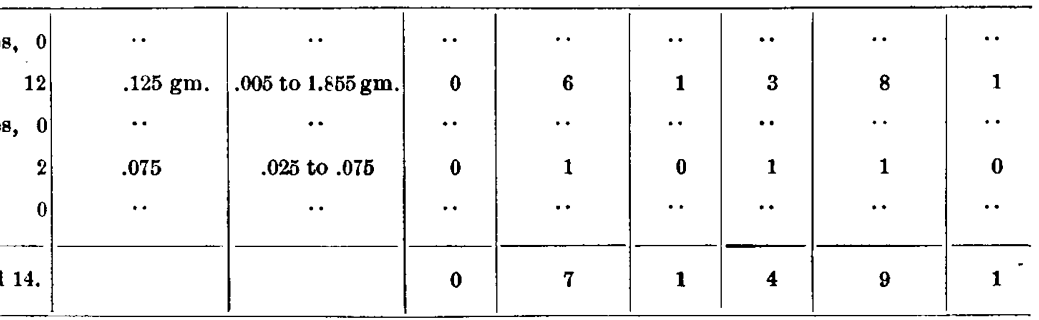

m Group I). - Patients Treated Firgt with Gold and Manganese, and Later with Hunter's Mod. B.

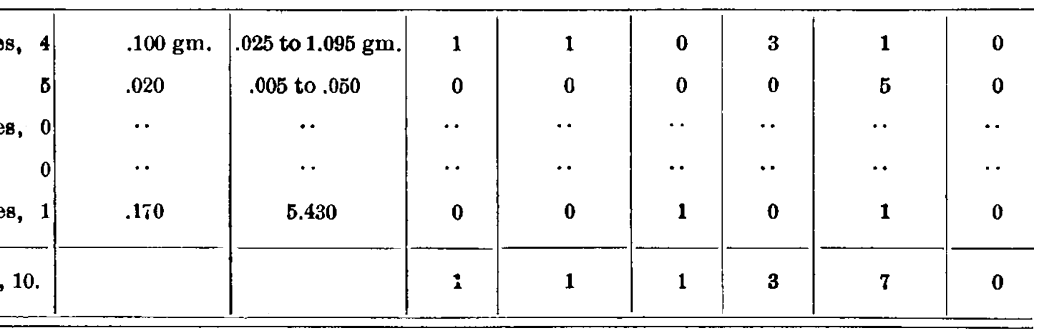

examination conld not be ascertained in every case, since some patients stopped their visits lexamination could be made. 
and although changes in the viscosity of the liquid owing to the temperature, the rapidity with which the drops are formed, and the shape of the material from which the drop falls largely govern the size, it was assumed that a drop from the original five-cubic-centimetre bottle, at about $70^{\circ} \mathrm{F}$, and forming at a speed of one in two and a half seconds, would be about the size usually obtained and probably intended by the quoted statement.

"Several careful weighings of ten-drop quantities of the liquid were made and the drops found to average 77 milligrammes each, and supposably contained onefiftieth of a grain (.0013) of U. S. P. strength of chloride of gold and sodium, and the same quantity of iodide of manganese, or $169 \mathrm{mg}$. of each in $10 \mathrm{gm}$. of injection.

"Ten grammes of the fluid were found to contain $.01737 \mathrm{gm}$. of metallic gold, equal to $.0535 \mathrm{gm}$. chloride of gold and sodium U.S. P., or about one-third the amount claimed. The liquid is composed mainly of glycerine, and contains free iodide, iodine in the form of iodate (also as iodide), chlorine, sodium, potassium and manganese.

"As the method of preparing the injection fluid recommended by $\mathrm{Dr}$. White was not made public, the following process has been devised, which yields a solution containing the ingredients he has reported as useful and of the strength he recommends. 'Take of

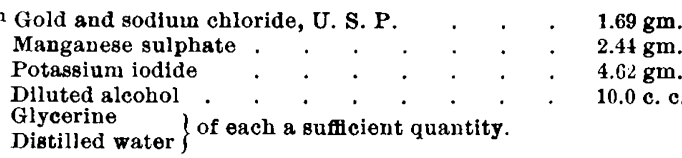

"Dissolve the gold and sodium chloride and one gramme of potassium iodide separately, in sufficient glycerine to make each solution weigh $25 \mathrm{gm}$. using ouly a very gentle heat, if any; when cold mix the two solutions. Dissolve the manganese sulphate in sufficient distilled water to make $10 \mathrm{c.}$ c., and $3.62 \mathrm{gm}$. of potassium iodide in sufficient diluted alcohol to make 10 c. c. ; mix the two solutions, and allow the containing vessel to stand in powdered ice for several hours, to thoroughly precipitate the potassium sulphate. Then decant $10 \mathrm{c}$. c. of the clear liquid into $40 \mathrm{gm}$. of glycerine; evaporate on a water bath until the total weight is reduced to $40 \mathrm{gm}$., and add glycerine to make $50 \mathrm{gm}$. Mix this solution with the solution of gold and sodium chloride, and keep in amber glass-stoppered bottles. Each $77 \mathrm{mg}$. (about one drop) contains the equivalent of $11^{3} \mathrm{mg}$. (one-fiftieth of a grain), of gold and sodium chloride and the same quantity of manganese iodide."

The injections were given in the forearm, upper arm and subclavicular region by au ordinary bypodermic syringe.

The solution prepared has been used in the manner advised by Dr. White, the initial dose usually being one drop (equal to one-twenty-fifth of a grain of the combined salts of gold and manganese) diluted with a few drops of a one-per-cent. solution of carbolic acid, and increased only as the constitutional disturbance remained at a minimum or ceased to appear after several doses.

In some patients the reaction appeared within two hours - as headache, backache and general malaise -

1 It is best to prepare this chemical or to use an article which is known to be of full strength, as market samples were found to con tain less gold than the labels indicated. lasting twenty-four to forty-eight hours; in others it was but little marked and disappeared within one or two hours after an injection.

The pulse has been found to be full and slow with subnormal temperature in some, while in others the pulse was increased in rate with a slight rise in temperature.

The local effect varied, in some the solution caused considerable inflammation, in others the injection was only marked by an induration and slight tenderness about the puncture. It seemed to depend upon the amount of loose connective-tissue present and the individual susceptibility to irritation.

Cough-mixtures, tonics and hygienic measures have also been made use of as opportunity permitted.

One thing was observed quite geverally, as noticed by another writer - within two weeks or thereabouts after beginning the injections, the appetite was increased, so much as to cause the remark of the patient to the effect.

The cough and expectoration in some were lessened, in others not appreciably affected. Night-sweats usually ceased. The general appearance and feelings of the patients improved, and they did not hesitate to declare themselves greatly benefited.

On making a physical examination of a patient after a few injections, the former condition of things may be somewhat aggravated. At such a time I have found the signs more marked, especially the auscultatory signs; later the results were variable.

The theory that the preparation of gold is eutrophic, while the manganese is anti-auæmic, seems to be well borne out. That an improvement can be secured in some cases I feel quite confident, but as to complete and permanent recoveries I am not so certain.

The exact way in which these injections affect the pulmonary tissue is more or less a matter of conjecture; nevertheless, the remedy appears to act as a tonic to the waning strength of the patient, also to cause a certain amount of irritation and inflammation around the foci of infection (as sometimes shown by bloody sputum after a large dose), which may eventually result in an encapsuling of the pathological portions, or their replacement by dense fibrous tissue.

However, be it as it may, the subcutaneous use of the chloride of gold and sodium with the iodide of manganese certainly improves the majority of patients subjectively; and if a cure does not result, they are at least in a most promising condition for treatment by modified tuberculin or its allied products. The strongest argument in favor of this treatment is the fact that when properly given no injury can be done, wbile there is great probability of an improvement if not a cure.

Appended is the full report of ten cases treated by gold and manganese, also the tabulated report of fiftyfour cases, - some having been treated with gold and manganese, and some with Hunter's modification B. (kindly furnished by Dr. Trudeau of Saranac Lake, N. Y.).

In regard to the latter method of treatment, I will briefly state, that while not obtaining the results of Dr. Trudeau, I found much in its favor to warrant a further trial.

I have also been using for several months Klebs' Tuberculocidin, and 80 far with considerable satisfaction.

In connection with this paper, I wish to thank Dr. 
E. M. Greene for his valuable services in the examination of sputum, and Dr. F. C. Cobb for laryngeal work, also Mr. Wm. C. Durkee, Ph.G., for most perfect chemical work, and Dr. E. O. Otis for kindly suggestions.

\section{ghedical Bragregifi.}

\section{REPORT ON MENTAL DISEASES.}

BY HENRY R. STEDMAN, M.D., BOBTON.

MENTAL DISTURBANCE IN EXOPTHALMIC GOITRE AND MYXOEDEMA.

HirschL ${ }^{1}$ considers that exopthalmic goitre is not often complicated with a psychosis. Mania is the ordinary intercurrent psychosis in a simple typical case. If another form comes on in the course of ex. opthalmic goitre, it is usually a complication of a coexisting or complicating neurastbenia, hysteria or alcoholism rather than of exopthalmic goitre itself. The psychical type of a patient with exopthalmic goitre is often between that of a healthy person and that of maniacal exaltation with signs of degeneration. This is very common, but not constant. It has equal value with the three cardinal symptoms, and can aid the diagnosis in doubtful cases. The explanation of complicating mania and this psychic type is not forced if we regard the disease as due to functional or anatomical disturbances in the medulla. These conditions can then be ascribed to vascular paresis of varying degree in the cortex. By thus localizing the disorder in the medulla we can better explain the less frequent pulse and the anxiety in psychical depressed states.

Clouston's ${ }^{2}$ study of the mental symptoms of myxœdema and the effect on them of the thyroid treatment is instinctive in showing that the contrast which marks their physical condition is equally evident in the mental states of these opposite affections of the thyroid. The general course of the mental disease in his cases was, tirst, slowness of mental action; secondly, emotional depression; next, irritahility, morbid suspicion, non-resistiveness to outward causes of disturbance and general loss of control or maniacal outbursts; then, enfeeblement, with some exaltation in some cases; and, lastly, lassitude, bebetude ending in a condition of mental negation just before death. Two pathological facts that have lately come under his observation in regard to the cerebral cortex have im. pressed him deeply with the possible recuperative capacity of the cortical structure. One.was a puerperal case of a few weeks standing - a curable case by every clinical standard - who died of maniacal exhaustion, and whose cortical cells were found in a state of marked and advauced degeneration, with spider cells and proliferated nuclei round the vessels and the neuroglia. If such degeneration is really curable, then we need not despair of recovery in many advanced cases of mental disease. The second fact is the actual cure of the prolonged mental enfeeblement of myxœedematous insanity by the thyroid treatment. He is convinced that we need not be hopeless of some day discovering remedies that will cure some of our cases of chronic melancholia, chronic mania and mild dementia, the pathological changes in whose brain cortex

\footnotetext{
1 Jahrbiichel für Psychiat., xii, 50.
}

he has often seen to be very similar to those found in one of his myxœdematous cases.

THE ALTERATIONB OF THE PERIPHERAL NeRVES IN GENERAL PARESIS.

G. Datto ${ }^{8}$ reports autopsies of eight cases of paresis in which he carefully examined by several methods (both fresh and after hardening) the peripheral nerves (various cranial nerves and nerves of the brachial and lumbar plexuses). His general results were as follows: The alterations met with were those of parenchymatous neuritis in different degrees. 'This was constant in all the observations, the variations in the intensity of the pathological process in each, it is suggested, were possibly in reiation to the intensity of the clinical symptoms. The cranial nerves were least affected in comparison with the others. The alterations were not systematic in their distribution, sometimes involving the motor, sometimes the sensory nerves; which fact in part explains, the author holds, the varied clinical syndromes of the disease. It also supports the view that we have in paresis a widely disseminated degenerative process affecting the most diverse organs of the nervous system.

Campbell, ${ }^{4}$ also, has made a careful study of the neuro-muscular changes in ten cases of general paralysis. He found the vagi extensively and strikingly diseased, more so- almost than any of the peripheral nerves, and decidedly more than any cranial nerve. It is impossible to attach too much importance to the remarkable siugling out of the vagi for such extreme degeneration in this disease. The changes in the mixed spinal nerves and their peripheral terminations appeared to be a combination of a parenchymatous degeneration and an interstitial or adventitious inflammation. This investigation confirms those of others in regard to the remarkable fact that the more peripheral the site examined in the mixed trunk the more extensive the degeneration will be found to be, and when the motor and sensory branches are reached it is more advanced and pronounced. The degree of degeneration of the spinal nerve roots was always considerable, but never extensive. The changes in the muscles do not, in the main, differ from those described in connection with other neurites. They are probably chiefly secondary effects of the nerve degeneration. It is extremely difficult to frame a distinct pathology for the neuro-muscular changes attendant on general paralysis, as there is so little knowledge of the precise nature of the pathogenic influence or factor which determines it ; still, taking these changes in general paralysis separately into consideration and comparing them with those in other varieties of multiple neuritis, we find that there exists a close resemblance from an anatomical standpoint, and there is further one group with which the changes in general paralysis can be pathogenetically compared, namely, the primary intrinsic toxamic; the secondary toxæmic, the purely toxic, the endemic, the rheumatic, and the cachectic or senile, being out of the question. This naturally does not refer to cases of alcoholic or syphilitic origin.

INEBRIATE ASYLUMS IN GERMANY AND SWITZERLAND.

Tilkowski ${ }^{5}$ visited and inspected five of these asylums : In Switzerland, Ellixon in Canton Zurich, Nüch-

8 El Pisani, xiv, p. 169.

4 Journal of Nervous and Mental Diseases, April, 1894

5 Jahrbüch. für Psych., xii, 1. 